\title{
Synthesis, morphology and rheology of core-shell silicone acrylic emulsion stabilized with polymerisable surfactant
}

\author{
H. H. Wang*, X. R. Li, G. Q. Fei, J. Mou \\ Key laboratory of Auxiliary Chemistry and Technology for Chemical Industry, Ministry of Education. Shaanxi University \\ of Science and Technology, Xi' an, 710021, P.R. China
}

Received 21 April 2010; accepted in revised form 2 July 2010

\begin{abstract}
Core-shell silicone acrylic emulsions with 3-methacryloxypropyl trimethoxysilane (MPTS) in the shell were prepared by seeded polymerization with the assistance of polymerisable maleate surfactant (MT). Fourier transform infrared (FT-IR) demonstrated the incorporation of polymerisable surfactant in copolymer. It was found that small amount of octadecyl acrylate was beneficial to emulsion stability with decreasing the particle size from 194.6 to $165.7 \mathrm{~nm}$. It was also found that the particle size increased from 165.7 to $242.9 \mathrm{~nm}$ with the increase of MPTS concentration. Furthermore, rheological measurement indicated that the emulsion was endowed with pseudoplasticity. At low shear rate, marginal reduction in viscosity was detected when MPTS concentration increased to $2 \%$, while great increase in viscosity was observed with higher MPTS concentration, the interaction force among emulsion particles became the predominant factor instead of particle size. In addition, better water resistance was observed when MT concentration was lower than 1.5\%, and MPTS concentration higher than $2 \%$. Moreover, surface roughness was increased with MPTS addition, the crosslinking among core and shell reconstructed the surface morphology of film.
\end{abstract}

Keywords: polymer synthesis, core-shell, rheology, morphology

\section{Introduction}

Polyarylate and poly(arylate-styrene) have been widely used in coatings, paints and adhesives so forth by virtue of excellent film-forming property, cohesiveness, gloss retention, and drying capacity. However, disadvantages in water resistance, mechanical property and weather resistance limit their applications [1-3]. In contrast, polysiloxane is endowed with excellent thermal stability, low surface energy, weather resistance, high flexibility and good biological compatibility owing to its unique structure [4-8]. In the past decades, research on silicone (or polysiloxane) modified polyarylate and poly(arylate-styrene), especially the product of core-shell structure, has attracted special attention of many researchers [9-12].
It is common knowledge that it is much easier to obtain core-shell morphology particles by seeded emulsion polymerization when the seed polymer is more hydrophobic than the shell polymer [13]. Therefore, normal core/shell morphology with hydrophobic polysiloxane (PSi) as the core and with hydrophilic polyacrylate (PA) as the shell can be easily formed, while it's difficult to obtain PA/ PSi core/shell particles in emulsion polymerization $[4,14]$. It is obvious that excellent surface properties can be created with small amount of PSi on the shell, which is beneficial to cost reduction. Under such circumstances, dispersion polymerization in alcoholic media is put forward and is demonstrated to be an effective way for the preparation of PA/PSi core/shell particles $[2,4,15]$. In addition, seeded 
polymerization [14-16] and sol-gel process [17] are also adopted to prepare core-shell particles with PSi as the shell. Zou et al. [16] points out that nanometer scale particles of latex can be successfully prepared by seeded polymerization induced by gamma rays. Kan et al. [10] state that perfect PA/ PSi core/shell particles can be synthesized when both the core and shell polymers are crosslinked, and 3-methacryloyloxyrpopyl trimethoxysilane has often been used as a crosslinking agent to form an intermediate layer between core and shell. However, ethanol and methanol have been utilized as medium instead of water in dispersion polymerization, which is detrimental to environment.

The main objective of this study is to prepare stable core-shell poly(butyl acrylate)-poly(styrene-butyl acrylate-3-methacryloxypropyl trimethoxysilane) (PBA-PSBM) emulsion particles with the assistance of polymerisable maleate surfactant with long branches, together with hydrophobic costabilizer octadecyl acrylate (ODA). Besides, seeded polymerization with water medium was adopted to prepare the emulsion. To date, no study about this system has been reported. It has been demonstrated that polymerisable surfactants exhibit an exceptionally high degree of incorporation into the emulsion polymer, protecting the emulsion from flocculation. Furthermore, the limited mobility of polymerisable surfactants avoids surface migration and the formation of hydrophilic domains in the films, resulting in greatly reduced process foaming, enhanced solvent resistance, improved adhesive and mechanical properties, and far less residual levels in effluent streams, as compared with nonreactive surfactants [22-27]. Anionic polymerisable maleate surfactants have already gotten extensive attention from a point of view of reactivity/copolymerization behavior [28, 29], and hydroxyethyl methacrylate on maleic anhydride is now available commercially [27]. However, since they are simply anionic, they don't provide steric stabilization and emulsions flocculate upon high shearing conditions or the addition of strong electrolytes or in freezing test. Maleate polymerisable surfactant with long branches was thereby synthesized which was able to provide electrostatic stabilization as well as steric stabilization.

In this research, seeded polymerization with water as medium was adopted to prepare PBA-PSBM core-shell emulsion, which was carried out under starved-feed addition of shell to prevent secondary nucleation and benefit to form core-shell structure [18-21]. And 3-methacryloxypropyl trimethoxysilane (MPTS) was introduced into the shell of the emulsion particles to endow them with selfcrosslinking properties in the film-forming process. Rheology, dynamic light scattering, transmission electron microscopy (TEM), as well as static contact angle and so on were performed to investigate the properties and morphology of core-shell particles.

\section{Experimental section}

\subsection{Materials}

3-Methacryloxypropyl trimethoxysilane (MPTS) (Wuhan Institute of Applied Technology, China) and nonyl phenol ethoxylate (TX-10) (Tianjin Kermel Chemical Reagent Co., Ltd, China) were used as received. Maleic anhydride (MAH), sodium bisulfite $\left(\mathrm{NaHSO}_{3}\right)$, potassium persulfate, and aqueous ammonia $\left(\mathrm{NH}_{3} \cdot \mathrm{H}_{2} \mathrm{O}\right)$ from Tianjin Dengfeng Chemical Reagent Co. (China) were all analytically pure grades, and were used without further purification. The analytical reagents butyl acrylate (BA), methacrylic acid (MAA), styrene (St) and octadecyl acrylate (ODA) were all purchased from Tianjing Chemical Reagent Factory (China) and were distilled under vacuum before use.

\subsection{Synthesis of polymerisable Surfactant (MT)}

1 mol maleic anhydride and $1 \mathrm{~mol}$ nonyl phenol ethoxylate were stirred in a melted state at $70^{\circ} \mathrm{C}$ for $3 \mathrm{~h}$. $\mathrm{NH}_{3} \cdot \mathrm{H}_{2} \mathrm{O}$ was then added dropwise to the reaction mixture to adjust $\mathrm{pH}$ value of reaction system to 6 and thereafter the temperature was maintained

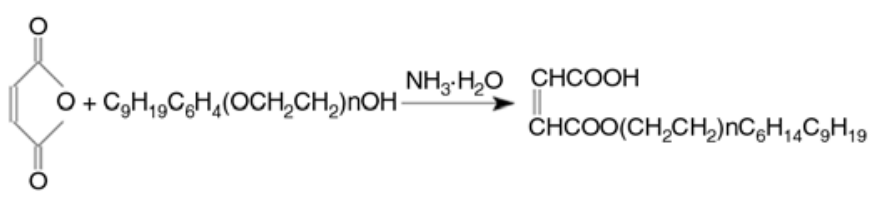

Figure 1. The chemical reaction of polymerisable surfactant 
in the range of $90-95^{\circ} \mathrm{C}$ until the solution becomes transparent. After $2 \mathrm{~h}$ of reaction, a kind of transparent solution with $35 \%$ solid content, that is, polymerisable surfactant (MT) was obtained by adding certain amount of distilled water. The reaction scheme was shown in Figure 1.

\subsection{Preparation of poly (BA-n-ODA) seed emulsion}

Polymerisable surfactant (MT) of different amounts and distilled water were introduced into a $250 \mathrm{ml}$ four-necked flask equipped with a reflux condenser, a mechanical stirrer and a digital thermometer. Then $16 \mathrm{~g} \mathrm{BA}, 0.5 \mathrm{~g} \mathrm{MAA}, 0.1 \mathrm{~g} \mathrm{NaHSO}_{3}$ and ODA of different amount were added into the flask. After additional $30 \mathrm{~min}$ equilibration at $70^{\circ} \mathrm{C}, 0.3 \mathrm{~g}$ potassium persulfate aqueous solution was dropped into the flask in $1 \mathrm{~h}$ and the final mixture was reacted at $70^{\circ} \mathrm{C}$ for additional $2 \mathrm{~h}$, the structure of seed copolymer was shown in Figure 2.

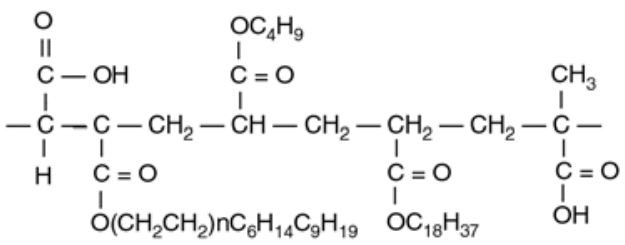

Figure 2. Structure of seed copolymer

\subsection{Seeded emulsion polymerization}

Using the particles prepared above as seeds, $0.2 \mathrm{~g}$ $\mathrm{NaHSO}_{3}$ and water was added into the seed emulsion. Then $16 \mathrm{~g} \mathrm{St}, 16 \mathrm{~g}$ BA, $1.0 \mathrm{~g}$ MAA and various amounts of MPTS in the dropping funnel was fed slowly into the flask under starved-feed addition, and simultaneously $0.5 \mathrm{~g}$ potassium persulfate aqueous solution was introduced into the flask in another dropping funnel with an appropriate dropping rate. The reaction was kept for another $2 \mathrm{~h}$ after the addition was completed. Then the obtained emulsion was cooled to room temperature and then

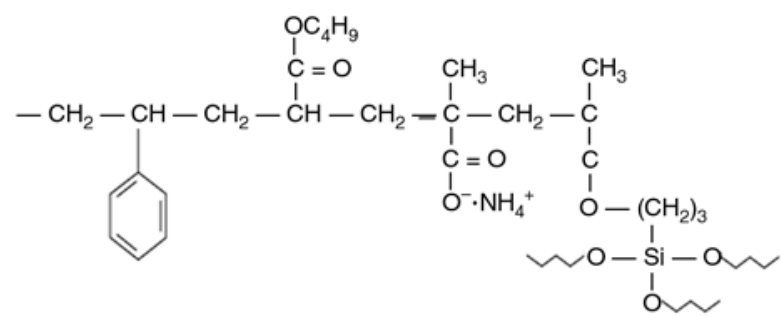

Figure 3. Structure of shell copolymer ammonia was added to adjust the $\mathrm{pH}$ value of emulsion in the range of 7-8. The structure of shell copolymer was shown in Figure 3.

\subsection{Structural analysis}

Fourier transform infrared (FT-IR) spectra were recorded on a Bruker Vector22 (Germany) Fourier Transform Infrared Spectrometer in the range from 4000 to $400 \mathrm{~cm}^{-1}$, by using polymer films. ${ }^{1} \mathrm{H}$ NMR and ${ }^{13} \mathrm{C}$ NMR were performed on a Bruker Avance $400 \mathrm{MHz}$ Spectrometer (Germany) with $\mathrm{D}_{2} \mathrm{O}$ as solvent.

\subsection{Stability of emulsions}

Centrifugal stability was determined by centrifugal method. Emulsions were filtered by 100 mesh sieve to remove impurities, and then $10 \mathrm{~g}$ emulsions were submitted to TDL-10B centrifuge (Shanghai Anting Scientific Instrument Factory, China) for $30 \mathrm{~min}$ at the speed of $3000 \mathrm{r} / \mathrm{min}$. Electrolyte-resistant stability was determined by adding electrolyte solutions to lattices. $5 \% \mathrm{CaCl}_{2}$ solution was added into the emulsion and kept for $48 \mathrm{~h}$, the ratio of coagulate was used as the standard for estimating emulsion stability. The ratio of coagulate $(\sigma)$ was calculated by Equation (1) [27]:

$$
\sigma[\%]=\frac{w_{c}}{w_{m}} \cdot 100
$$

where $w_{c}$ is the weight of dried coagulates and $w_{m}$ is total weight of all monomer. The larger the values of $\sigma$, the worse the stabilities are.

\subsection{Particle size and morphology measurement}

The particle size of the emulsions was analyzed by Ver 2.14 dynamic light scattering (Zeta-plus, Brookhaven Instruments Co. US). Its morphology was observed by Japanese Hitachi S570 transmission electron microscope (TEM) with phosphotungstic acid as staining agent.

\subsection{Rheology measurement}

The rheological properties of emulsions were analyzed in an American TA Instrument ${ }^{\circledR}$ AR2000ex Rheometer. Steady state flow tests (shear rate from 
0.01 to $100 \mathrm{~s}^{-1}$ ) were carried out at $25^{\circ} \mathrm{C}$ using DIN concentric cylinders geometry. Sample period: 0:00:01, percentage tolerance: 5.0, consecutive within tolerance: 3, maximum point time: 0:01:00.

\subsection{Atomic force microscopy (AFM)}

The films were prepared by spin coating in a freshly cleaved glass plate. The film was dried for a few minutes in air at room temperature before observation by Japanese Seiko SPI3800N/SPA 400 atomic force microscopy (AFM). All measurements were made in the contact mode.

\subsection{Water resistance}

Membranes were cut into circular disks by using a sharp-edged stainless steel die with inner diameter of $20 \mathrm{~mm}$. The samples were dried in vacuum oven for $24 \mathrm{~h}$ to determine their dry weight $(W)$. Absorption of the membranes was determined by immersing the membranes in a beaker of medium for $24 \mathrm{~h}$. After wiping off the surface water with a piece of filter paper, its weight $\left(W_{1}\right)$ was determined. The absorption (WS) of the films was calculated by Equation (2):

$W S=\frac{W_{1}-W}{W} \cdot 100$

\subsection{Contact angle}

Contact angles were measured with a JJC-1 contact angle goniometer (Chengde Tester factory, P.R. China) at $27^{\circ} \mathrm{C}$ with water as medium, and the results reported are the mean values of 5 replicates.

\section{Results and discussion}

\subsection{Structure analysis}

The ${ }^{1} \mathrm{H}$ NMR and ${ }^{13} \mathrm{C}$ NMR spectra of TX-10 and MT were presented in Figure 4 and Figure 5, respectively. As shown in Figure 4, the characteristic peaks of the protons in the $-\mathrm{CH}=\mathrm{CH}-$ group $(\delta=6.17 \mathrm{ppm})$ in ${ }^{1} \mathrm{H}$ NMR spectrum of MT demonstrate the reaction between maleic anhydride and TX-10, which is further certified by ${ }^{13} \mathrm{C}$ NMR spectra. As shown in Figure 5, the characteristic peaks at $\delta=170.61 \mathrm{ppm}$ can be assigned to carbons in $-\mathrm{C}=\mathrm{O}$ group of $\mathrm{MT}$, while peaks at $\delta=$

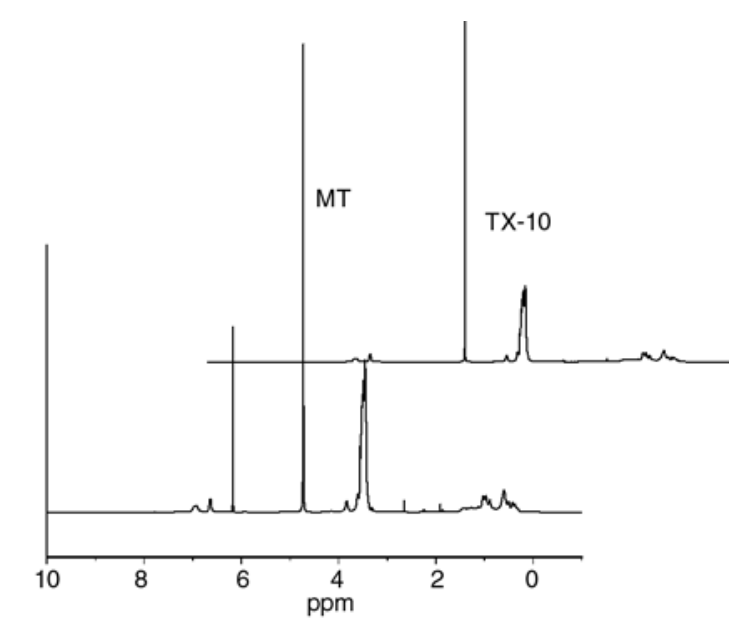

Figure 4. ${ }^{1} \mathrm{H}$ NMR spectrum of TX-10 and MT

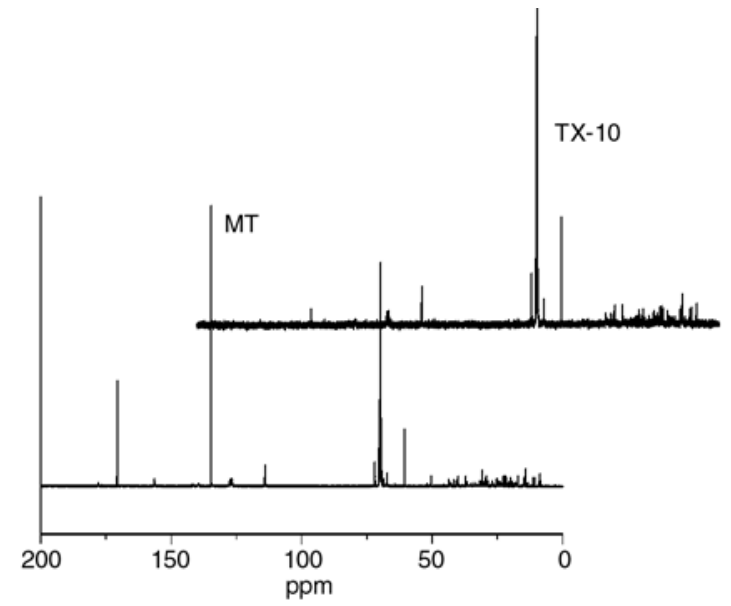

Figure 5. ${ }^{13} \mathrm{C}$ NMR spectrum of TX-10 and MT

$134.62 \mathrm{ppm}$ results from $-\mathrm{C}=\mathrm{C}-$ group in $\mathrm{MT}$. Therefore, it can be concluded that polymerisable surfactant was successfully prepared.

The FT-IR spectra of MT polymerisable surfactant and core/shell polymer were shown in Figure 6. The existence of $-\mathrm{COOH}$ in MT could be confirmed by the broad peak at $3482 \mathrm{~cm}^{-1},-\mathrm{C}=\mathrm{O}$ stretching vibration at $1733 \mathrm{~cm}^{-1}$ and $-\mathrm{C}-\mathrm{O}$ stretching vibration at $1248 \mathrm{~cm}^{-1}$. While the characteristic peak at $3482 \mathrm{~cm}^{-1}$ disappears in coreshell polymer, and the characteristic peak corresponding to $-\mathrm{C}=\mathrm{O}$ at $1732 \mathrm{~cm}^{-1}$ gets stronger, the main reason can be attributed to the transfer from carboxylic acid to carboxylic salt.

It is also found that the characteristic peaks corresponding to the stretching vibration of $\mathrm{CH}=\mathrm{CH}$ in the region of 1650 to $1500 \mathrm{~cm}^{-1}$ in FTIR spectrum of MT are absent in the spectrum of core-shell polymer, as well as the bending vibration of $\mathrm{CH}=\mathrm{CH}$ at $948 \mathrm{~cm}^{-1}$, demonstrating the incorporation of MT in copolymer material. 


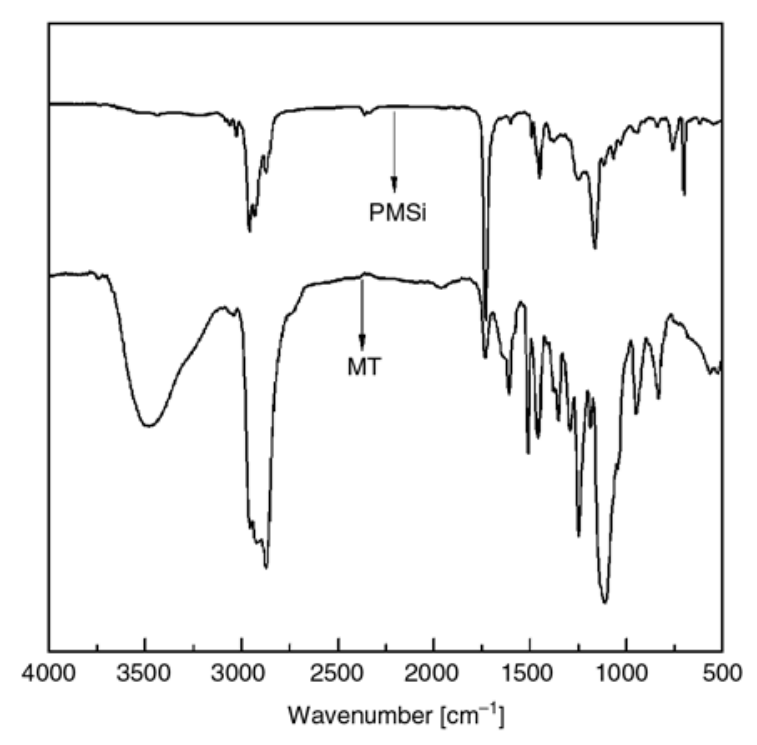

Figure 6. FT-IR spectra of polymerisable surfactant (MT) and core/shell polymer

\subsection{Effects of polymerisable surfactant}

Figure 7 showed effects of MT concentration on emulsion stability without any ODA and MPTS addition. It can be seen that the coagulate ratio decreased with the increase of MT and kept almost invariable when MT concentration was greater than $2 \%$ (based on total weight of monomer, the following were the same), without displaying decreasing trend with higher MT concentration. It has been reported that redundant surfactant would homopolymerize to form waterborne polymer chain that can cause flocculation, resulting in lower stability with higher surfactant concentration [29]. While for MT, it is difficult for homopolymerization to take place among MT molecules owing to its long branches, which can stabilize the emulsion through electros-

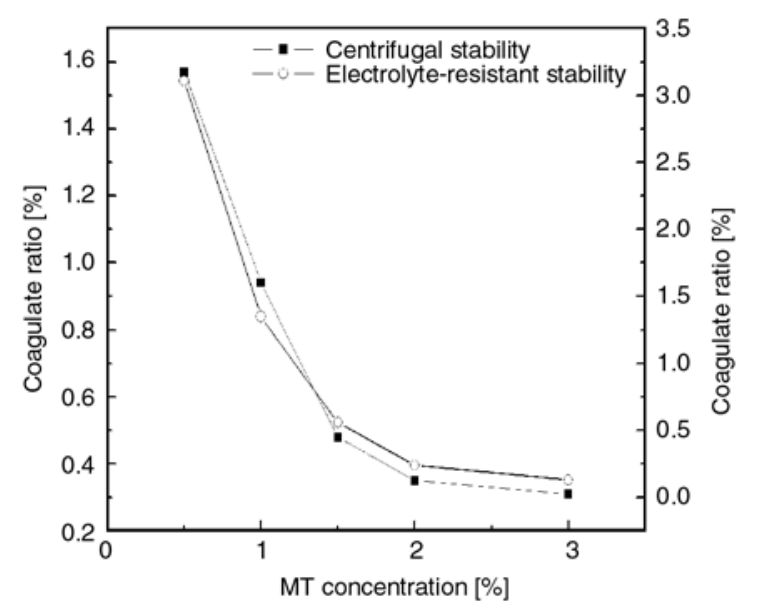

Figure 7. Emulsion stability with different MT concentration teric effects. $1.5 \%$ MT was utilized for preparation of the following samples.

\subsection{Effects of octadecyl acrylate (ODA)}

Octadecyl acrylate, as a kind of vinyl monomer possessing long and highly hydrophobic side chain, can improve micelle solubilization to a certain extent, to date some block copolymers containing polyoctadecyl segments have been reported to be used as stabilizers [30]. Effects of ODA concentration on emulsion stability with $1.5 \% \mathrm{MT}$ and $0 \%$ MPTS were presented in Figure 8. It was found that ODA concentration should be neither too high nor too low. With the increase of ODA concentration, the emulsion stability increased accordingly, but decreased greatly when ODA concentration was more than $0.5 \%$. It is stated that the destabilization of the droplets can be efficiently slowed down by an addition of a hydrophobic agent to the dispersed phase which counteracts the droplet pressure or Laplace pressure of the droplet [31]. The hydrophobe can act as an osmotic stabilizing agent. However, the hydrophobicity of copolymer chain was increased simultaneously, which will weaken the emulsion stability and result in the increase of coagulate ratio.

At the same time, effects of ODA concentration on particle size were analyzed, as shown in Figure 9. The average particle size decreased from 194.6 to $165.7 \mathrm{~nm}$ with $0.5 \%$ ODA addition, and increased to $187.0 \mathrm{~nm}$ with further increase of ODA concentration to $1.0 \%$. The main reason is that ODA can act as a kind of costabilizer during copolymerization, which can reduce the diffusion of the monomer

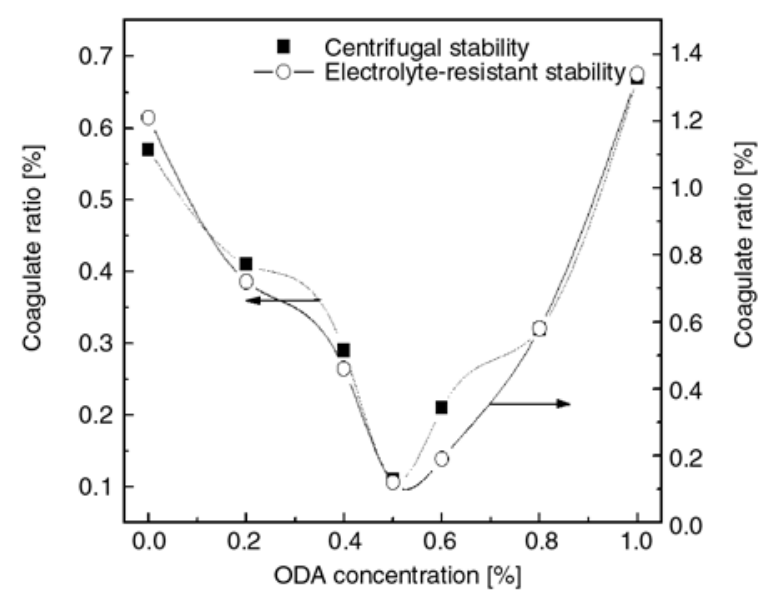

Figure 8. Emulsion stability with different ODA concentration 


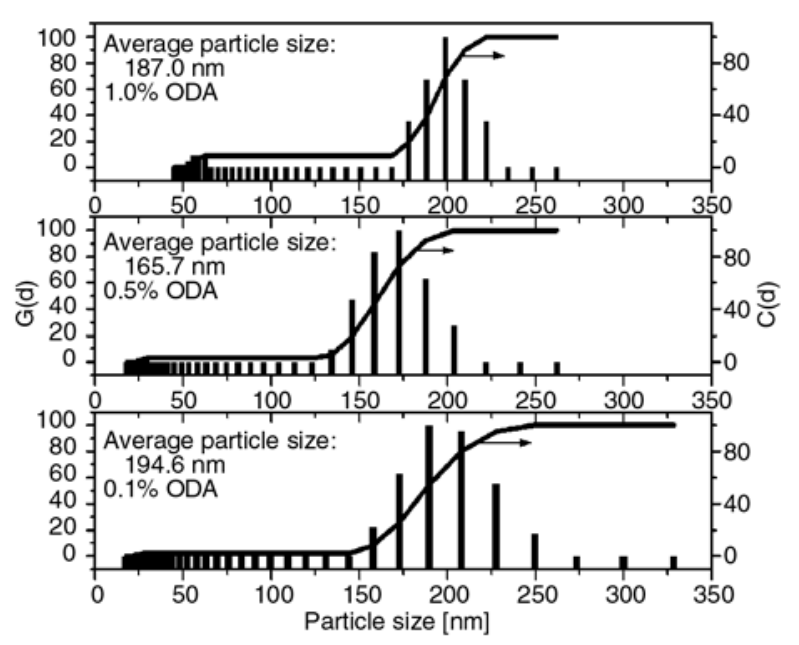

Figure 9. Particle size of emulsion with different ODA concentration

from smaller droplets to the larger one [32], resulting in the shrinkage of droplets with lower ODA concentration. Whereas the size of droplets with higher hydrophobe concentration will increase till the chemical potential in all droplets is equilibrated [31]. The following samples in this paper were prepared with $0.5 \%$ ODA.

\subsection{Effect of 3-methacryloxypropyl trimethoxysilane (MPTS)}

MPTS is a kind of important copolymerization monomer with self-crosslinking property in the film-forming process, which plays an important role in the improvement of water resistance and mechanical property. However, the $\mathrm{Si}-\mathrm{O}-\mathrm{C}$ bond in MPTS molecules is much easier to hydrolyze and condense both in acidic media and alkali media, which would bring instability to the emul-

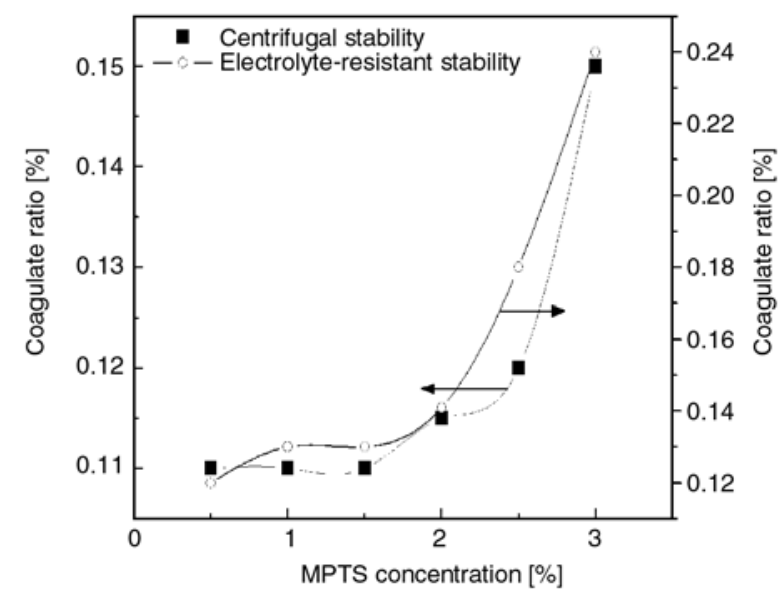

Figure 10. Effect of MPTS concentration on emulsion stability

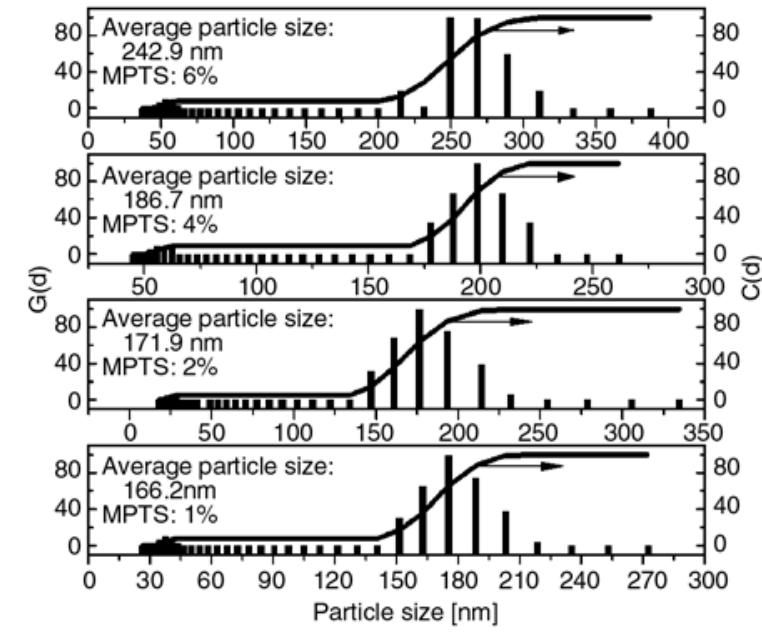

Figure 11. Particle size of the core/shell emulsion with different MPTS concentration

sions [1]. Effects of MPTS concentration on emulsion stability with $1.5 \%$ MT and $0.5 \%$ ODA were presented in Figure 10. The emulsion kept stable when MPTS concentration was less than $4.0 \%$, but the amount of precipitate increased greatly beyond this point, indicating that increasing $\mathrm{Si}-\mathrm{OH}$ concentration resulted in the instability of emulsions.

The average particle size of the emulsions with different MPTS concentration by DLS was shown in Figure 11. A monotonic increase in particle size from 165.7 to $242.9 \mathrm{~nm}$ with MPTS addition was observed. With the increase of MPTS concentration, the polymer chain length increased due to the crosslinking, causing a lower growing particle number, as a result, the diameter of particles become larger.

TEM micrographs of the seed particles and core/ shell particles with 4\% MPTS were shown in Figure 12 and Figure 13, respectively. As shown in Figure 12, all the PBA seed particles were spherical in shape with white color. It is also evident that the

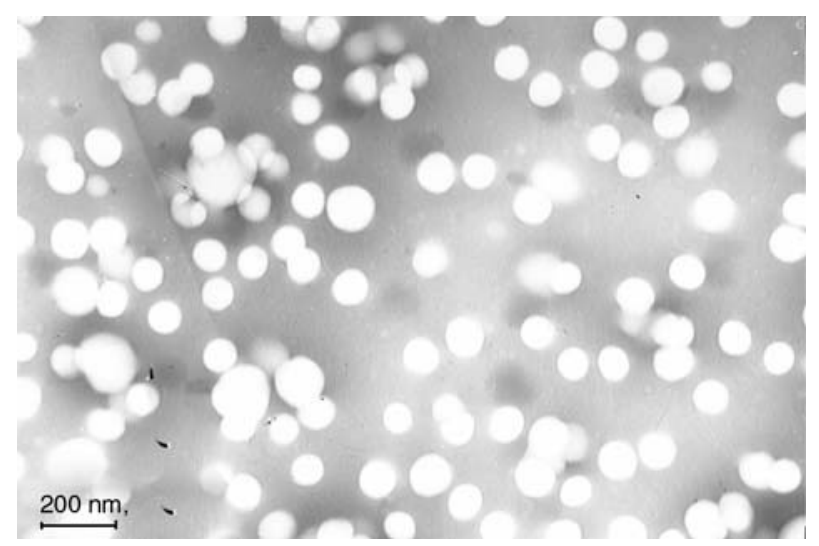

Figure 12. TEM micrograph of seed emulsion $(\times 50000)$ 


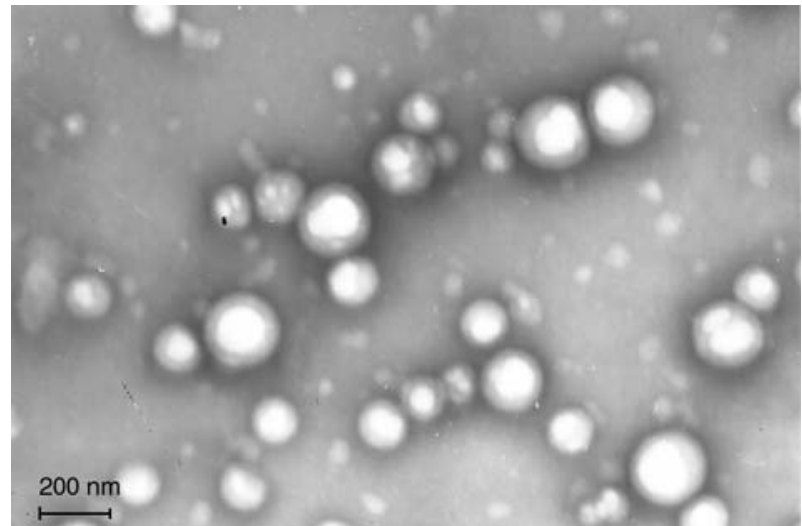

Figure 13. TEM micrograph of core/shell emulsion with $4 \%$ MPTS $(\times 50000)$

deposition of black PSBM shell onto the white seed particles is uniform without any voids, further indicating expectant PBA-PSBM core-shell structure has been formed in this study. And covalent bond between core and shell formed by MPTS can contribute to this phenomenon, too.

\subsection{Rheological properties of core-shell emulsion}

The viscosities and shear stress of core/shell emulsions with different MPTS concentration were plotted as a function of shear rate in Figure 14 and Figure 15, respectively. It was striking that the viscosity decreased greatly with the increase of shear rate at the start and then gradually to a constant level, indicating that the core/shell emulsions were endowed with pseudoplasticity.

At low shear rate, marginal reduction in viscosity and shear stress was detected when MPTS concen-

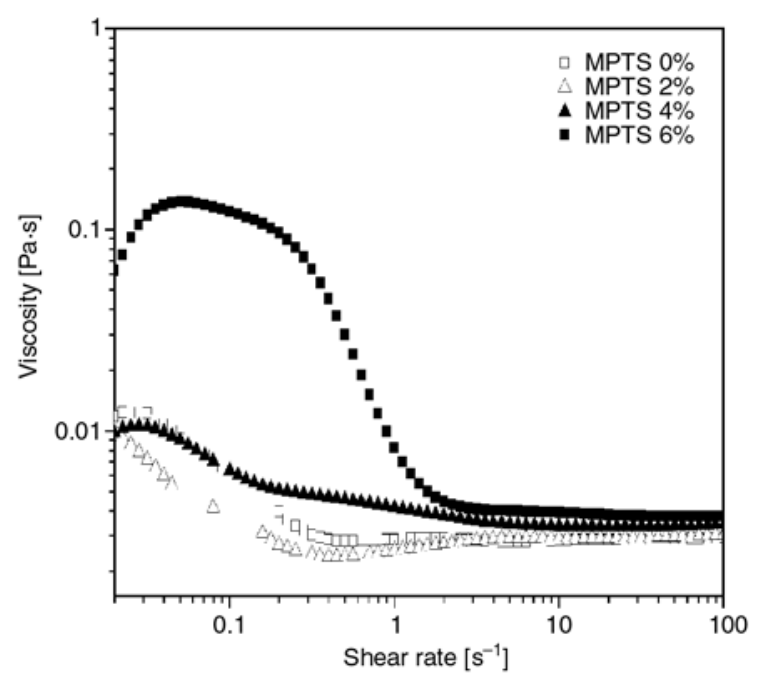

Figure 14. Viscosity versus shear rate curves with variation in MPTS concentration

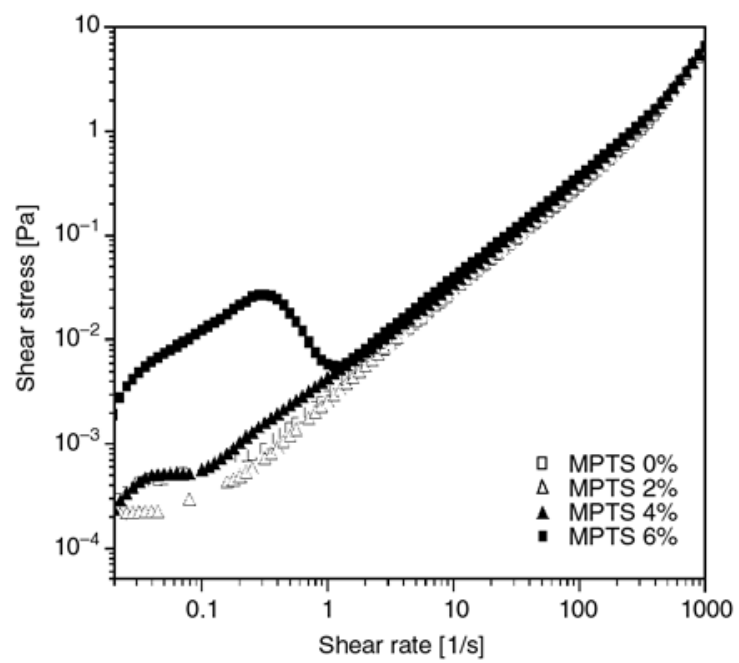

Figure 15. Shear stress versus shear rate curves with variation in MPTS concentration

tration increased to $2 \%$, which could be ascribed to the increase of particle size. A smaller particle size generally increased the low shear viscosity due to colloidal interactions. However, a period of plateau was observed, as well as great increase in viscosity when MPTS concentration was greater than $4 \%$, indicating interaction force among latex particles became the predominant factor influencing the viscosity instead of particle size.

While when shear rate was greater than $1 \mathrm{~s}^{-1}$, the viscosity became constant with shear rate and simultaneously increased slightly with MPTS concentration, since viscosity is often independent of particle size at higher shear rates, as hydrodynamic forces dominate.

\subsection{AFM analysis}

AFM was used to examine the film surface and to measure its surface topography. The AFM pictures of core/shell emulsion films with 0 and 4\% MPTS were shown in Figure 16 and Figure 17, respectively. The surface of the film with 0\% MPTS showed a lower root-mean-square roughness value compared with the film with $4 \%$ MPTS, indicating increased surface roughness with MPTS addition. Therefore it was evident that the cross-linking between core and shell induced by MPTS reconstructed the surface morphology of the film. And the root mean square roughness induced by entanglements and penetration of molecular chain increased in the process of film-forming, demonstrating the increase of particle size, which was 

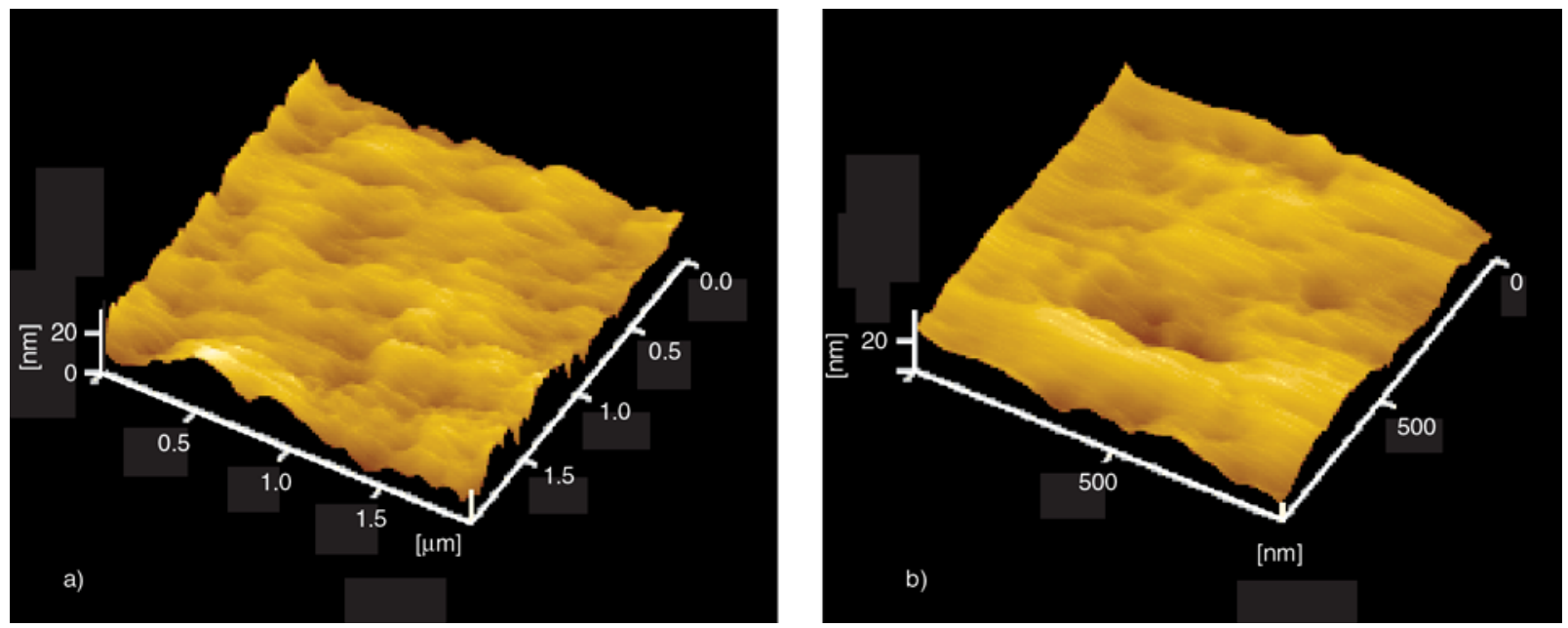

Figure 16. AFM images of the film with $0.0 \%$ MPTS
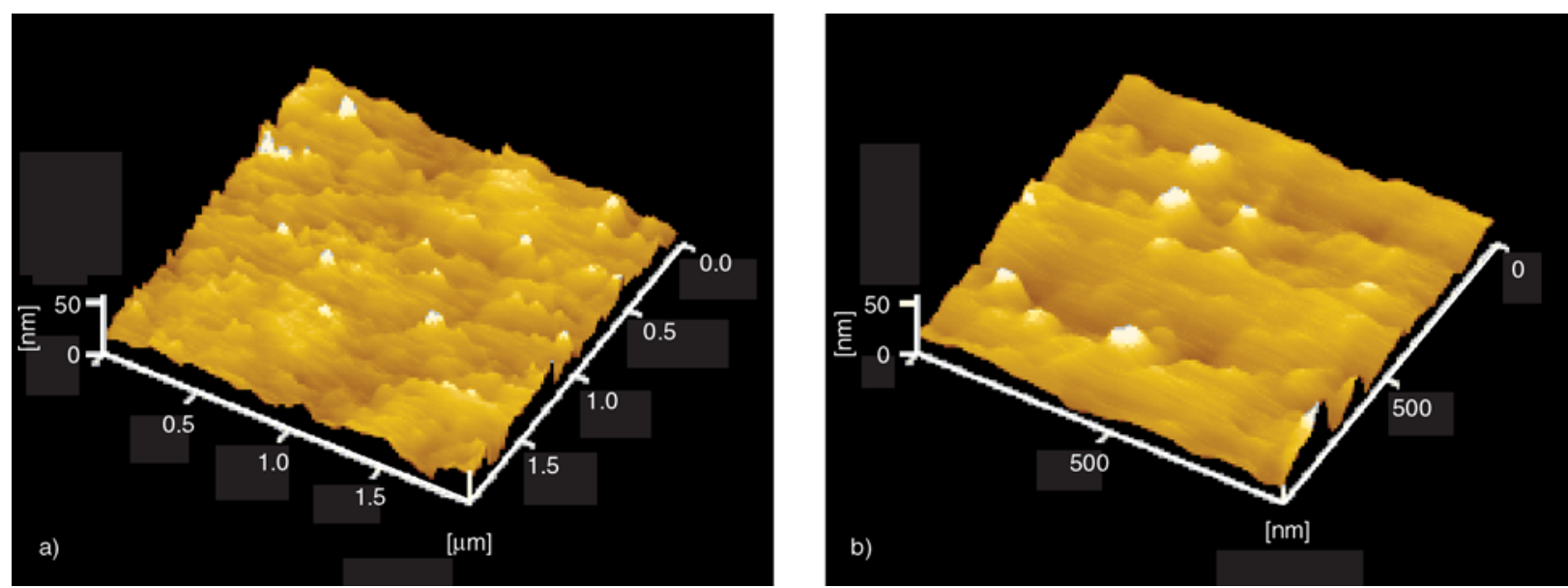

Figure 17. AFM images of the film with $4 \%$ MPTS

coincident with the aforementioned results shown in Figure 11.

\subsection{Water resistance analysis}

Figure 18 presented effects of MT concentration on the water absorption and contact angle of the films. As MT concentration increased from 0.5 to $1.5 \%$, the water absorption decreased from 8.33 to $5.26 \%$, and contact angle increased from 54 to $79^{\circ}$. However, when MT concentration exceeded $1.5 \%$, the water absorption increased from 5.26 to $22.51 \%$, contact angle decreased from 79 to $64^{\circ}$. The particle size was relative smaller with higher MT concentration, resulting in better particle packing and thus leading to the decrease of water absorption. On the other hand, hydrophilic groups greatly increased with the further increase of MT concentration, so the MT concentration can't be too high, or else water resistance will be weakened.

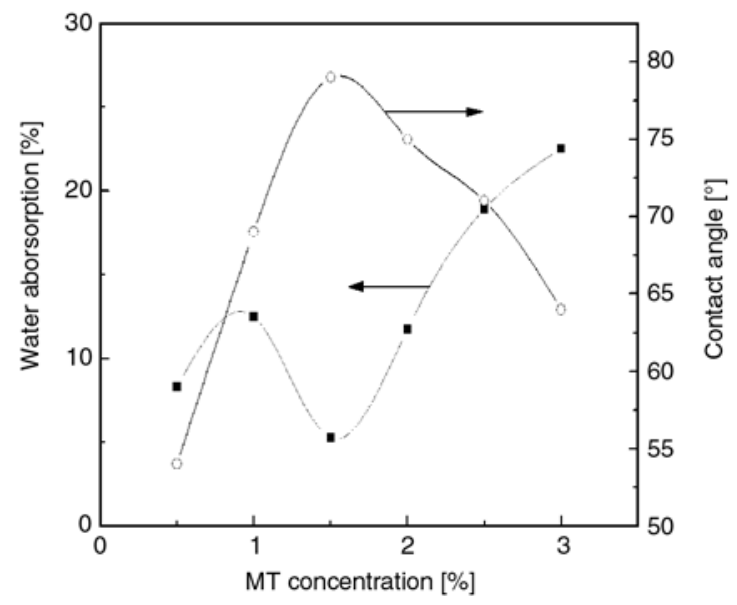

Figure 18. Effect of MT concentration on water absorption and contact angle of the films

Effects of MPTS concentration on the water absorption of the films were also investigated, as shown in Figure 19. It was found that the water absorption decreased with MPTS addition, providing a powerful proof that water resistance was 


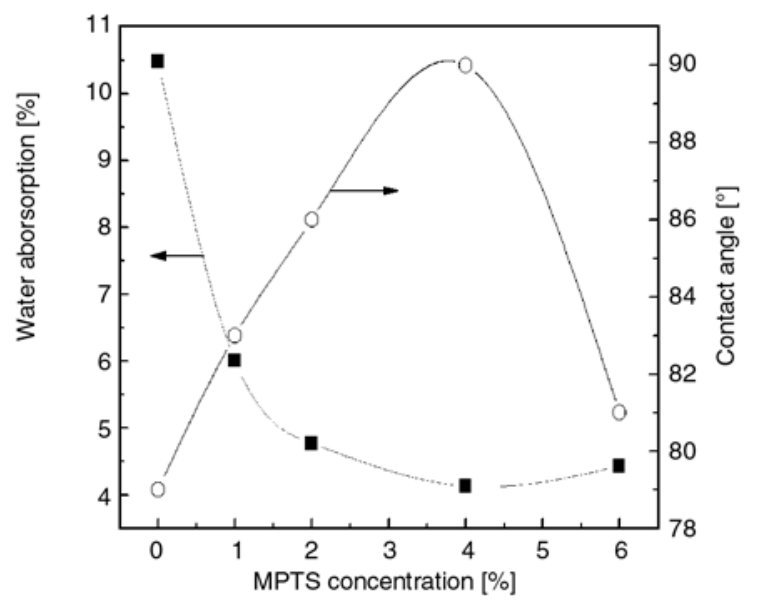

Figure 19. Effect of MPTS concentration on water absorption and contact angle of the films

enhanced by MPTS addition. It was also found that the surface contact angle increased with MPTS addition, indicating hydrophobic surface can be obtained by introducing MPTS into the shell. Conventionally, hydrophobic surfaces have been produced mainly in two ways. One is to create a rough structure on a hydrophobic surface, and the other is to modify a rough surface by materials with low surface free energy [17]. Therefore, the relative rougher surface created by MPTS (as shown in Figure 17) can be considered as another motivation for hydrophobicity improvement in addition to increased hydrophobic moieties and crosslinking degree in the shell.

\section{Conclusions}

Core-shell silicone acrylic emulsions with 3methacryloxypropyl trimethoxysilane (MPTS) in the shell were successfully prepared by seeded polymerization with the assistance of polymerizable maleate surfactant. Emulsions were endowed with excellent stability when MT concentration was higher than $2.0 \%$ and ODA concentration was $0.5 \%$. The particle size decreased from 194.6 to $165.7 \mathrm{~nm}$ with $0.5 \%$ ODA addition, and increased to $187.0 \mathrm{~nm}$ with further addition of ODA. It was also found that the emulsion kept stable when MPTS concentration was less than $4.0 \%$, and the particle size increased from 165.7 to $242.9 \mathrm{~nm}$. Furthermore, emulsions were endowed with pseudoplasticity. At low shear rate, marginal reduction in viscosity was detected when increased to $2 \%$, how- ever, great increase in viscosity was observed with higher MPTS concentration, the interaction force among emulsion particles become the predominant factor instead of particle size. On the contrary, the viscosity became constant when shear rate was greater than $1 \mathrm{~s}^{-1}$. In addition, the surface roughness was increased with MPTS addition, the crosslinking among core and shell reconstructed the surface morphology of film. And water resistance could be improved with MPTS addition.

\section{Acknowledgements}

The authors express sincere thanks to the National Natural Science Foundation of China (No. 20876093), Natural Science Foundation of Shaanxi Province of China (No. 2007E118), Natural Science Foundation of Shaanxi Province of China (No. 2009JQ2004) and Foundation of Shaanxi University of Science \& Technology (ZX08-07, BJ09-18) for financial support.

\section{References}

[1] Guo T. Y., Chen X., Song M. D., Zhang B. H.: Preparation and properties of core [poly(styrene- $n$-butyl acrylate)]-shell [poly(styrene-methyl methacrylatevinyl triethoxide silane)] structured latex particles with self-crosslinking characteristics. Journal of Applied Polymer Science, 100, 1824-1830 (2006). DOI: 10.1002/app.23081

[2] Cao S. S., Liu B. L., Deng X. B., Li S. J.: Core/shell particles containing 3-(methacryloxypropyl)-trimethoxysilane in the shell: Synthesis, characterization, and application. Macromolecular Bioscience, 5, 669-676 (2005).

DOI: $10.1002 / \mathrm{mabi} .200500011$

[3] Bauer F., Gläsel H. J., Decker U., Ernst H., Freyer A., Hartmann E., Sauerland V., Mehnert R.: Trialkoxysilane grafting onto nanoparticles for the preparation of clear coat polyacrylate systems with excellent scratch performance. Progress in Organic Coatings, 47, 147153 (2003). DOI: $\underline{10.1016 / \mathrm{S} 0300-9440(03) 00117-6}$

[4] Liu B. L., Deng X. B., Cao S. S., Li S. J., Luo R.: Preparation and characterization of core/shell particles with siloxane in the shell. Applied Surface Science, 252, 2235-2241 (2006). DOI: $10.1016 /$ j.apsusc. 2005.03.223

[5] Lin F., Song M., He Z., Zhang T.: Synthesis and structural characterization of methacrylic acid/octadecyl acrylate-graft-poly(methylhydrosiloxane) by hydrosilylation. Journal of Applied Polymer Science, 107, 3773-3780 (2008).

DOI: $10.1002 / a p p .27487$ 
[6] Turner J. S., Cheng Y. L.: Preparation of PDMSPMAA interpenetrating polymer network membranes using the monomer immersion method. Macromolecules, 33, 3714-3718 (2000).

DOI: $10.1021 / \mathrm{ma991873 \textrm {k }}$

[7] Liu B. L., Zhang B. T., Cao S. S., Deng X. B., Hou X., Chen H.: Preparation of the stable core-shell latex particles containing organic-siloxane in the shell. Progress in Organic Coatings, 61, 21-27 (2008).

DOI: $10.1016 /$ j.porgcoat.2007.08.008

[8] Zaioncz S., Dahmouche K., Paranhos C. M., Gil R. A. S., Soares B. G.: Relationships between nanostructure and dynamic-mechanical properties of epoxy network containing PMMA-modified silsesquioxane. Express Polymer Letters, 3, 340-351 (2009).

DOI: $10.3144 /$ expresspolymlett.2009.43

[9] Kan C. Y., Kong X. Z., Yuan Q., Liu D. S.: Morphological prediction and its application to the synthesis of polyacrylate/polysiloxane core/shell latex particles. Journal of Applied Polymer Science, 80, 2251-2258 (2001).

DOI: $10.1002 / a p p .1329$

[10] Kan C. Y., Liu D. S., Kong X. Z., Zhu X. L.: Study on the preparation and properties of styrene-butyl acrylate-silicone copolymer lattices. Journal of Applied Polymer Science, 82, 3194-3200 (2001).

DOI: $\underline{10.1002 / a p p .2178}$

[11] He W-D., Pan C-Y.: Influence of reaction between second monomer and vinyl group of seed polysiloxane on seeded emulsion polymerization. Journal of Applied Polymer Science, 80, 2752-2758 (2001). DOI: $\underline{10.1002 / a p p .1390}$

[12] Zhang T., Xi K., Chen H., Yu X.: Synthesis and properties of self-crosslinkable polyurethane-urea with silsesquioxane formation. Journal of Applied Polymer Science, 91, 190-195 (2004). DOI: $10.1002 / a p p .13212$

[13] Kong X. Z., Kan C. Y., Yuan Q.: Preparation of polyacrylate-polysiloxane core-shell latex particles. Polymers for Advanced Technologies, 7, 888-890 (1996). DOI: 10.1002/(SICI)1099-1581(199612)7:12<888::

\section{AID-PAT598>3.0.CO;2-5}

[14] Kan C. Y., Kong X. Z., Yuan Q., Liu D. S.: Morphological prediction and its application to the synthesis of polyacrylate/polysiloxane core/shell latex particles. Journal of Applied Polymer Science, 80, 2251-2258 (2001). DOI: $10.1002 /$ app.1329

[15] Cao S. S., Liu B. L., Deng X. B., Luo R., Chen H. L.: A novel approach for the preparation of acrylate-siloxane particles with core-shell structure. Polymer International, 56, 357-363 (2007).

DOI: $10.1002 /$ pi.2149
[16] Zou M. X., Zhang Z. C., He W. D., Ge X. W., Fan F.: Preparation and characterization of core-shell polystyrene-polydimethylsiloxane particles by seeded polymerization. Polymer International, 53, 1033-1039 (2004).

DOI: $\underline{10.1002 / \mathrm{pi} .1470}$

[17] Xin L., Zhong X.: Preparation and characterization of micron-sized polystyrene/polysiloxane core/shell particles. Colloid and Polymer Science, 284, 1062-1066 (2006).

DOI: $10.1007 / \mathrm{s} 00396-006-1482-\mathrm{y}$

[18] Dreher W. R., Jarret W. L., Urban M. W.: Stable nonspherical fluorine-containing colloidal dispersions: Synthesis and film formation. Macromolecules, 38, 2205-2212 (2005).

DOI: $10.1021 / \mathrm{ma} 0479161$

[19] Ha J-W., Park I. J., Lee S-B., Kim D-K.: Preparation and characterization of core-shell particles containing perfluoroalkyl acrylate in the shell. Macromolecules, 35, 6811-6818 (2002).

DOI: $\underline{10.1021 / \mathrm{ma} 011692 \mathrm{u}}$

[20] Ha J-W., Park I. J., Lee S-B.: Hydrophobicity and sliding behavior of liquid droplets on the fluorinated latex films. Macromolecules, 38, 736-744 (2005). DOI: $\underline{10.1021 / \mathrm{ma} 0488764}$

[21] Cui X. J., Zhong S. L., Gao Y., Wang H. Y.: Preparation and characterization of emulsifier-free core-shell interpenetrating polymer network-fluorinated polyacrylate latex particles. Colloids and Surfaces A: Physicochemical and Engineering Aspects, 324, 14-21 (2008).

DOI: $10.1016 /$ j.colsurfa.2008.03.018

[22] Soula O., Guyot A.: Styrenic surfmer in emulsion copolymerization of acrylic monomers: I. Synthesis and characterization of polymerizable surfactants. Langmuir, 15, 7956-7962 (1999). DOI: $10.1021 / 1 \mathrm{la} 9817009$

[23] Texter J.: Reaction and synthesis in surfactants systems. Marcel Dekker, NewYork (2001).

[24] Thenoz F., Soula O., Guyot A.: Reactive surfactants in heterophase polymerization. XXV. Core-shell lattices stabilized with a mixture of maleic anionic and styrenic nonionic surfactants. Journal of Polymer Science Part A: Polymer Chemistry, 37, 2251-2262 (1999).

DOI: 10.1002/(SICI) 1099-0518(19990701)37:13

$$
\leq 2251: \text { :AID-POLA40>3.0.CO;2-R }
$$

[25] Chen S-A., Chang H-S.: Kinetic and mechanism of emulsifier-free emulsion polymerization: Styrene/surface active ionic comonomer system. Journal of Polymer Science: Polymer Chemistry Edition, 23, 26152630 (1985). DOI: $\underline{10.1002 / \text { pol.1985.170231005 }}$

[26] Onodera S., Yamamoto S., Tamai T., Takahashi H.: Reactive emulsifiers for polymerization of vinyl compounds. Japanese Patent JP 06239908, Japan (1994). 
[27] Guyot A.: Recent progress in reactive surfactants in emulsion polymerization. Macromolecular Symposia, 179, 105-132 (2002).

DOI: $10.1002 / 1521-3900(200203) 179: 1<105:: A I D-$ MASY105>3.0.CO;2-7

[28] Schoonbrood H. A. S., Unzué M. J., Beck O-J., Asua J. M.: Reactive surfactants in heterophase polymerization. 7. Emulsion copolymerization mechanism involving three anionic polymerizable surfactants (surfmers) with styrene-butyl acrylate-acrylic acid. Macromolecules, 30, 6024-6033 (1997).

DOI: $\underline{10.1021 / \mathrm{ma} 9701447}$

[29] Schoonbrood H. A. S., Asua J. M.: Reactive surfactants in heterophase polymerization. 9. Optimum surfmer behavior in emulsion polymerization. Macromolecules, 30, 6304-6041 (1997).

DOI: $10.1021 / \mathrm{ma9701494}$
[30] Qin S. H., Saget J., Pyun J., Jia S. J., Kowalewski T., Matyjaszewski K.: Synthesis of block, statistical, and gradient copolymers from octadecyl (meth)acrylates using atom transfer radical polymerization. Macromolecules, 36, 8969-8977 (2003).

DOI: $\underline{10.1021 / \mathrm{ma} 0349985}$

[31] Antonietti M., Landfester K.: Polyreactions in miniemulsions. Progress in Polymer Science, 27, 689-757 (2002).

DOI: 10.1016/S0079-6700(01)00051-X

[32] Quzineb K., Graillat C., Mckenna T. F.: Study of compartmentalization in the polymerization of miniemulsions of styrene and butyl methacrylate. Journal of Applied Polymer Science, 91, 115-121 (2004). DOI: 10.1002/app.13181 